\title{
Características da informação valiosa: o case de uma indústria de bebidas
}

Alcançar ou manter uma colocação vantajosa no mercado, diante de um cenário em contínua mudança, é realidade para poucos. Destacam-se as organizaçõe que sabem que a informação de qualidade pode trazer vantagens significativas, se bem explorada e gerenciada. Por acreditar nisso, este trabalho buscou identificar a percepção dos gerentes regionais de vendas quanto à eficiência e à eficácia dos principais relatórios disponibilizados pela Área de Informações de uma indústria de bebidas, por meio de uma pesquisa aplicada, com abordagem quantitativa e qualitativa, com uma etapa descritiva, e bibliográfica, documental e participante quanto aos procedimentos técnicos. Para a coleta de dados, foram aplicados questionários eletrônicos, com onze respondentes. Para dar apoio/embasamento teórico, fundamentou-se o estudo com diversos autores da área de Gestão da Informação. Os resultados mostraram que na percepção dos gerentes regionais de vendas, o "Relatório A" é eficiente e eficaz e o "Relatório B" não é eficiente e eficaz, sendo necessária avaliação e possível reestruturação, evidenciando a falta de gestão da informação, principalmente no "Relatório B". Após análise e discussão dos resultados, sugere-se validar formas de conferência antes da disponibilização das informações; utilizar recursos tecnológicos para otimização do tempo e levantar as necessidades dos usuários.

Palavras-chave: Informação valiosa; Eficiência; Eficácia; Gestão da informação; Sistemas de informação.

\section{Characteristics of valuable information: the case of a beverage industry}

Reaching or maintaining an advantageous placing in the market is a reality for few ones, facing a scenario of continuous change. The organizations that know the information of quality can bring meaningful advantages, if it is well explored and managed, are highlighted. With this belief, this study aimed to identify the perception of the regional sales managers regarded to the efficiency and effectiveness of the main reports provided by the Information Section of a beverage industry, through an applied research, with quantitative and qualitative approach, with a descriptive stage, bibliographic, documental and participant in relation to the technical procedures. For the data collection, electronic questionnaires were applied, with eleven respondents. In order to have theoretical approach, several authors from the Information Management area were mentioned. The results presented that, according to the regional sales managers, "Report A" is efficient and effective and "Report B" is not efficient and effective, making an evaluation and possible restructuring necessary, showing the lack of information management, mainly in "Report B". After the analysis and discussion of results, it is suggested to validate forms of conference before sharing the information and also use technological resources to optimize time and find out the users' needs.

Keywords: Valuable information; Efficiency; Effectiveness; Information management; Information systems.

Topic: Sistemas e Tecnologia da Informação

Reviewed anonymously in the process of blind peer.
Received: 25/01/2017

Approved: 24/03/2017

\section{Andre Kohl}

Universidade de Santa Cruz do Sul, Brasil http://lattes.cnpq.br/4410944819672175 andre kohl2001@yahoo.com.br

\section{Referencing this:}

KOHL, A.. Características da informação valiosa: o case de uma indústria de bebidas. Revista Brasileira de Administração Científica, v.8, n.1, p.270-281, 2017. DOI: http://doi.org/10.6008/SPC2179$\underline{684 X .2017 .001 .0020}$ 


\section{INTRODUÇÃO}

As organizações encontram-se inseridas em um ambiente dinâmico e competitivo, associado a mudanças tecnológicas, econômicas, sociais e culturais. Um dos desafios, diante desse cenário, está na gestão da informação, fundamental para que se possa prever, compreender e responder às mudanças, objetivando alcançar ou manter uma posição favorável no mercado. Com o passar do tempo, torna-se visível que o número de informações disponíveis em qualquer área cresce significativamente, levando as empresas a buscarem ferramentas de processamento e capital humano qualificado para transformar dados em informações. A entrega de informações confiáveis e no prazo desejado é essencial para a tomada de decisão.

Para Beal (2012), a disponibilização de informações confiáveis é tudo, pois as mesmas podem fortalecer ou mesmo causar grandes perdas às organizações. Nesse contexto, observa-se a importância da avaliação das características da informação valiosa, pois garante a eficiência e a eficácia das informações disponibilizadas, e permite, também, o desenvolvimento de estratégias, melhorias em processos, atendimento de necessidades, visando a diferenciais competitivos.

Ciente da importância das informações para a organização como um todo e do crescimento da busca pelas mesmas, as organizações precisam ter seus processos decisórios e operacionais alimentados com informações eficientes e eficazes, ou seja, de qualidade e adaptadas às necessidades do negócio. Diante do exposto, buscou-se identificar: qual a percepção dos gerentes regionais de vendas quanto à eficiência e à eficácia dos principais relatórios disponibilizados pela Área de Informações de uma indústria de bebidas?

Este estudo foi desenvolvido em uma indústria de bebidas, envolvendo a Área de Informações, o Gerente Estratégico de Mercado e onze gerentes regionais de vendas. A relação dos gerentes regionais de vendas, contendo o nome, o telefone, o e-mail e a região de atuação, e a relação dos relatórios, contendo o nome, a frequência de disponibilização e os destinatários, foram fornecidas pela Área de Informações da indústria de bebidas, contudo, não constam neste trabalho a fim de preservar o sigilo das informações.

Cabe salientar que este estudo avaliou somente os dois principais relatórios quanto às características da informação valiosa, por abrangerem informações gerenciais, e os demais complementarem essas informações. Da mesma forma, considerou somente os gerentes regionais de vendas, visando a obter uma percepção gerencial. Quanto à característica da informação valiosa 'econômica', não foi mensurada, pois as informações estão disponíveis na indústria de bebidas, não havendo custos para obtê-las.

O objetivo geral deste estudo buscou identificar a percepção dos gerentes regionais de vendas quanto à eficiência e à eficácia dos principais relatórios disponibilizados pela Área de Informações de uma indústria de bebidas. Quanto aos objetivos específicos, visaram identificar o perfil dos gerentes regionais de vendas; identificar a quantidade e os principais relatórios disponibilizados aos gerentes regionais de vendas pela Área de Informações de uma indústria de bebidas; avaliar se os principais relatórios têm as características da informação valiosa, através da escala Lickert, segundo a percepção dos gerentes regionais de vendas e propor melhorias aos principais relatórios disponibilizados aos gerentes regionais de vendas.

A necessidade emergente de avaliar as informações disponibilizadas justifica a importância deste estudo para as organizações, que expostas a desafios e oportunidades, buscam ser eficazes e eficientes, 
atendendo às demandas por informações. Para a Área de Informações da empresa, os resultados permitem melhor análise das informações disponibilizadas e servem como subsídio para possíveis melhorias.

Para a instituição de ensino, o trabalho serve para avaliar se o conhecimento disponibilizado durante o curso foi aplicado e, aos acadêmicos, como fonte de consulta para outras pesquisas. E, para Beal (2012), que atua na Área de Informações da indústria de bebidas, permite o estudo mais detalhado sobre gestão da informação, visando a propor melhorias, além de complementar o processo de ensino e aprendizagem.

\section{REVISÃO TEÓRICA}

\section{Dado, informação, conhecimento e competência}

Este capítulo apresenta a fundamentação teórica sobre gestão da informação, partindo da definição de dado, informação, conhecimento e competência; do valor da informação para as organizações; sistemas de informação; tipos de sistemas de informação; gestão da informação e suas etapas; eficiência e eficácia e características da informação valiosa.

A definição de dado, informação, conhecimento e competência se faz necessária por tratar de conceitos fortemente relacionados e, por muitas vezes, confundidos. Conforme Beal (2012) e Audy et al. (2007), dados são registros ou fatos em sua forma primária. Bio (2008) complementa que dado é "[...] um elemento da informação (um conjunto de letras ou dígitos), que, tomado isoladamente, não transmite nenhum conhecimento, ou seja, não contém um significado intrínseco". Já informação "[...] são dados concatenados, que passaram por um processo de transformação, cuja forma e conteúdo são aprimorados para um uso específico" (AUDY et al., 2007). Complementa Beal (2012) que a informação surge da organização ou combinação de dados.

O conhecimento possui forte ligação com a informação, pois consiste em entender as informações e identificar como elas podem ser úteis para o processo. Audy et al. (2007) acrescenta que o conhecimento envolve "[...] uma combinação de instintos, ideias, informações, regras e procedimentos que guiam ações e decisões". Relacionando aos conceitos apresentados, cabe salientar a competência, pois é por meio dela que ocorre o aprimoramento de conhecimentos, habilidades e atitudes. Assim, alguém é competente quando constitui, articula e mobiliza valores, conhecimentos e habilidades para uma atuação transformadora (HELAL et al., 2009).

\section{O valor da informação para as organizações}

A informação dentro das organizações deve assumir o caráter de suporte e ser utilizada como fator de apoio à decisão, de produção, de sinergia e determinante de comportamento, assim, precisa ser de qualidade, relevante, precisa e clara (BEAL, 2012). Ainda na opinião de Beal (2012), a informação é necessária no processo de tomada de decisão, pois permite que as escolhas sejam feitas com maior segurança e no momento certo. Audy et al. (2007) salienta que os gestores precisam de informações sobre a organização e o ambiente externo da empresa, com vistas a identificar ameaças e oportunidades, criando um cenário para 
uma resposta eficaz e competitiva. No processo de produção, a informação é necessária, mais especificamente na criação e introdução de novos produtos, bens ou serviços no mercado (BEAL, 2012).

Quanto ao fator de sinergia, Beal (2012) explica que as unidades da cadeia produtiva precisam, além de apresentar bom desempenho, estar alinhadas. Desta forma, o fluxo de informações é necessário para que haja sinergia entre as unidades. Por fim, mas não menos importante, a informação também é fator determinante de comportamento, seja ele interno ou externo. Conforme Beal (2012), internamente, as informações servirão de subsídio para que os indivíduos busquem os objetivos corporativos, e externamente, objetivarão influenciar as pessoas para o alcance dos objetivos organizacionais.

\section{Sistemas de informação}

Diante da necessidade das organizações por informações que auxiliem na tomada de decisão, no controle das operações, na análise de problemas e na criação de novos produtos ou serviços, um sistema de informação é fundamental. Conforme Audy et al. (2007), "o objetivo geral dos sistemas de informação é disponibilizar para a organização as informações necessárias para que ela atue em um determinado ambiente".

Beal (2012) destaca que sistema de informação "[...] é um conjunto de elementos ou componentes que interagem para atingir objetivos". Beal (2012) acrescenta que os sistemas têm entradas, instrumentos de processamento, saídas e feedback. Por meio da utilização de sistemas de informação, de acordo com Audy, Andrade e Cidral (2007), as organizações melhoram sua gestão, automatizam os processos, integram áreas, clientes e fornecedores, disponibilizam informações confiáveis para todos os níveis organizacionais, evitam perda de informações, eliminam retrabalhos e reduzem custos.

\section{Tipos de sistemas de informação}

Muitas são as formas de classificar os sistemas de informação, contudo, as classificações pela finalidade de uso e pelo nível organizacional são as mais aceitas, conforme Audy et al. (2007). Como este trabalho tem como objetivo identificar a percepção dos gerentes regionais de vendas, abordou-se os sistemas utilizados no nível tático, ou seja, o Sistema de Informação Gerencial (SIG) e o Sistema de Apoio à Decisão (SAD).

Para Audy et al. (2007), o Sistema de Informação Gerencial (SIG) atende "em grande parte os gerentes de nível tático da organização na forma de relatórios que apresentam indicadores sobre o desempenho de uma determinada área". O Sistema de Apoio à Decisão (SAD) auxilia na disponibilização de informações para tomar decisões semiestruturadas.

Estes sistemas integram dados dos sistemas de informação gerencial, de processamento de transações e de fontes externas (AUDY et al., 2007). Além da integração com outros sistemas, o mesmo permite "realizar análises e simulações como forma de comparar o impacto de diferentes decisões" (AUDY et al., 2007). 


\section{Gestão da informação e suas etapas}

A gestão da informação assegura o valor estratégico da informação, pois é um processo que consiste nas atividades de identificação, busca, classificação, tratamento, armazenamento e disseminação de informações. Gestão da informação, para Beal (2012), é um processo que envolve muitas etapas, desde identificação de necessidades e requisitos, até obtenção, tratamento, distribuição, uso, armazenamento e descarte de informações. Abordando mais especificamente as etapas da gestão da informação, a identificação de necessidades e requisitos, segundo Beal (2012), consiste em entender o que o indivíduo ou grupo precisa, é compreender qual informação deve ser buscada.

Em seguida, temos a etapa de obtenção dos dados que, de acordo com Audy et al. (2007), podem ser extraídos de fontes de origem ou de bancos de dados. A próxima etapa é a de tratamento, momento em que os dados obtidos são tratados, e conforme Beal (2012), ocorre a "[...] organização, formatação, estruturação, classificação, análise, síntese e apresentação, com o propósito de torná-la mais acessível e fácil de localizar pelos usuários".

Em seguida, vem a distribuição, etapa na qual as informações já tratadas são disponibilizadas aos usuários. O uso, por sua vez, "possibilita a combinação de informações e surgimento de novos conhecimentos, que podem voltar a alimentar o ciclo da informação corporativa, num processo contínuo de aprendizado e crescimento" (BEAL, 2012).

Quanto ao armazenamento, as informações devem ser facilmente localizadas, seguindo um padrão, e devem poder ser utilizadas diversas vezes. Acrescenta Audy et al. (2007) que o armazenamento precisa ser feito de modo seletivo, para não entulhar os gestores com configurações muito amplas que dificultem seu acesso. Por fim, a etapa de descarte, que deve ocorrer quando a informação se torna obsoleta ou quando não é mais utilizada pela organização.

\section{Eficiência e eficácia}

A eficiência objetiva mais rapidez nas operações e menos custos, contudo, com as constantes mudanças sociais, políticas, econômicas e tecnológicas, as organizações estão se importando também com a eficácia, ou seja, com a otimização de resultados. Para Bio (2008), "eficiência diz respeito a método, a modo certo de fazer as coisas. É definida pela relação entre volumes produzidos/recursos consumidos". Compartilhando do mesmo entendimento, Beal (2012) salienta que eficiência é a "relação entre os produtos gerados por um processo e os custos dos insumos empregados".

Desta forma, organizações eficientes avaliam os recursos envolvidos no processo para produzir um volume de produtos, bens ou serviços com o menor custo. Quanto à eficácia, Bio (2008) destaca que "diz respeito a resultados, a produtos decorrentes de uma atividade qualquer". Ainda na opinião do autor, "a eficácia é definida pela relação entre resultados pretendidos/resultados obtidos" (BIO, 2008). Diante do exposto, as organizações são eficazes quando conseguem atender quantitativamente e qualitativamente as necessidades que surgem. 


\section{Características da informação valiosa}

As características da informação valiosa visam a maior segurança à ideia central da informação, visto que a mesma pode ser facilmente manipulada e seu sentido modificado. A informação deve gerar novos conhecimentos e auxiliar no processo de tomada de decisão. Para que as informações sejam eficientes e eficazes, algumas características precisam ser atribuídas a elas, conforme quadro 1.

Quadro 1: Características da informação valiosa.

\begin{tabular}{|l|l|}
\hline \multicolumn{1}{|c|}{$\begin{array}{c}\text { Características da } \\
\text { informação valiosa }\end{array}$} & \multicolumn{1}{c|}{ Descrição das características da informação valiosa } \\
\hline Precisa/Exata & $\begin{array}{l}\text { A informação não deve ter erro. Cuidado, pois informaçães incorretas lançadas na entrada seguem } \\
\text { durante o processamento e saída. }\end{array}$ \\
\hline Completa & $\begin{array}{l}\text { Informações completas contêm todos os fatos relevantes para o processo em análise. Cuidado; a } \\
\text { falta de um registro produzirá uma informação incompleta. }\end{array}$ \\
\hline Econômica & Necessário avaliar o valor da informação e o custo para obtê-la. \\
\hline Flexível & $\begin{array}{l}\text { A informação flexível corresponde à maneira como é armazenada, possibilitando análises diferentes, } \\
\text { ou seja, podem ser utilizadas para diferentes propósitos, para mais de um objetivo. }\end{array}$ \\
\hline Confiável & $\begin{array}{l}\text { Uma informação confiável é aquela na qual se pode acreditar. Cuidado com os dados de origem e os } \\
\text { métodos de coleta de dados. }\end{array}$ \\
\hline Relevante & São informações importantes para os tomadores de decisão. \\
\hline Clara/Simples & $\begin{array}{l}\text { A informação deve ser clara e simples. A complexidade/excesso pode dificultar a tomada de decisão } \\
\text { e tornar-se desnecessária. }\end{array}$ \\
\hline Pontual/Veloz & $\begin{array}{l}\text { As informações devem ser entregues quando necessárias. Se forem para uma reunião e entregarem } \\
\text { depois, não terão utilidade. }\end{array}$ \\
\hline Verificável & $\begin{array}{l}\text { A informação deve permitir uma verificação por parte do tomador de decisão, para que sua validade } \\
\text { seja assegurada. }\end{array}$ \\
\hline Acessível & $\begin{array}{l}\text { A informação precisa estar ao alcance de todos que precisam utilizá-la, de forma fácil e } \\
\text { descomplicada, no formato e momento certos. }\end{array}$ \\
\hline Segura & $\begin{array}{l}\text { A informação precisa estar disponível a quem interessa e precisa estar segura para evitar o acesso } \\
\text { por parte de usuários não autorizados. }\end{array}$ \\
\hline
\end{tabular}

Fonte: Audy et al. (2007); Reynolds et al. (2011).

Diante do exposto, cabe salientar que a boa gestão da informação evita que informações importantes para o sucesso das organizações não sejam exploradas, além de controlar o volume de informações e de recursos utilizados para a obtenção de informações sem utilidade, tornando o processo de gestão da informação mais eficiente e eficaz.

\section{METODOLOGIA}

\section{Caracterização da pesquisa}

Esse capítulo apresenta os procedimentos metodológicos utilizados, visando a atender aos objetivos que motivaram o estudo. Segundo Marconi et al. (2010), trata-se de um conjunto de atividades que auxiliam a traçar o caminho a ser percorrido. Trata-se de uma pesquisa aplicada, pois buscou identificar a percepção dos gerentes regionais de vendas quanto à eficiência e à eficácia dos principais relatórios. Para Gil (2010), pesquisas aplicadas são "pesquisas voltadas à aquisição de conhecimentos com vista à aplicação numa situação específica".

Este estudo apresentou uma etapa quantitativa e uma qualitativa, além de ser caracterizado também como descritivo. A pesquisa quantitativa é uma "metodologia de pesquisa que procura quantificar os dados e, geralmente, aplica alguma forma de análise estatística" (MALHOTRA, 2012). O mesmo autor explica que a pesquisa descritiva é um "tipo de pesquisa conclusiva que tem como principal objetivo a descrição de algo - 
normalmente características ou funções do mercado" (MALHOTRA, 2012). Assim, aplicam-se a este trabalho, pois o mesmo buscou avaliar se os principais relatórios têm as características da informação valiosa, através da escala Lickert. Quanto à pesquisa qualitativa, Roesch (2013) destaca que "é apropriada para a avaliação formativa, quando se trata de melhorar a efetividade de um programa, plano, [...]", aplicando-se a este estudo, pois melhorias foram sugeridas aos relatórios pesquisados.

\section{Delimitação da pesquisa}

Para Malhotra (2006), a população-alvo é um conjunto de elementos que possui a informação e que é objeto do estudo do pesquisador. Vergara (2006) acrescenta que o universo corresponde à população da pesquisa e a amostra é uma parte do universo, escolhida por meio de critérios de representatividade. Este estudo buscou abranger toda a população-alvo, logo, não utilizou amostra e sim, censo, os onze gerentes regionais de vendas da indústria de bebidas foram pesquisados, visando a obter uma percepção gerencial a respeito dos principais relatórios. Segundo Marconi e Lakatos (2010, p. 206), censo "abrange a totalidade dos componentes do universo".

\section{Técnicas e instrumentos de coleta de dados}

Utilizou-se a pesquisa bibliográfica para a elaboração da fundamentação teórica e dos procedimentos metodológicos, por meio de livros que abordam temas pertinentes ao estudo. Conforme Gil (2011), levantamento bibliográfico refere-se a publicações em livros, artigos científicos, monografias, dissertações, entre outros. A pesquisa documental apoiou-se em fontes documentais disponibilizadas pela Área de Informações da indústria de bebidas, como a relação dos onze gerentes regionais de vendas e a relação de relatórios disponibilizados para eles. Para Roesch (2013), a pesquisa documental é constituída por documentos como relatórios anuais da organização, materiais utilizados, políticas de marketing, entre outros.

Como a autora trabalha na Área de Informações da indústria de bebidas e participou das reuniões para identificar a quantidade de relatórios disponibilizados e os principais, utilizou-se a pesquisa participante. Segundo Gil (2010), a pesquisa participante pode seguir um plano de ação, contudo, também pode ser feita por meio de reuniões e debates. Para a coleta de dados, adotou-se o questionário eletrônico aplicado aos onze gerentes regionais de vendas, sem identificação dos mesmos. A pesquisa ocorreu de 30 de agosto a 09 de setembro de 2016. O questionário apresentou uma etapa estruturada, com questões fechadas, e outra não estruturada, com uma questão aberta.

Segundo Gil (2010), o questionário deve traduzir os objetivos específicos da pesquisa, em questões bem redigidas. Acrescenta Botelho et al. (2006) que o questionário eletrônico é acessado por computador, na internet, ou enviado por e-mail, ou seja, é distribuído por meio eletrônico. Vergara (2010) explica que "no questionário aberto, as respostas livres são dadas pelos respondentes; no fechado, o respondente faz escolhas, ou pondera, diante de alternativas apresentadas". 
Antes da aplicação do questionário, aplicou-se pré-testes a dois respondentes, quais sejam, o gestor da Área de Informações e o Gerente Estratégico de Mercado, sendo que ambos não apresentaram dificuldades em responder, não havendo necessidade de ajustes. Para Malhotra (2006), pré-teste é um "teste do questionário com uma pequena amostra de entrevistados com o objetivo de identificar e eliminar problemas potenciais".

Os resultados dos questionários foram apurados por meio da escala Likert; que segundo Malhotra (2012, p. 222), “[...] é fácil de construir e de aplicar, e os entrevistados entendem rapidamente como utilizar a escala, [...]". Este trabalho considerou as dimensões "nunca a sempre" entre 5 (cinco) níveis de pontuação, onde 1 corresponde a "nunca" e 5 a "sempre", para que os respondentes identificassem nos principais relatórios o nível de cada característica da informação valiosa.

\section{Técnicas de análise de dados}

Análise dos dados, para Vergara (2010), é uma técnica que visa a identificar o que está sendo dito a respeito do tema. Acrescentam Marconi e Lakatos (2010) que após a coleta de dados, os mesmos necessitam ser tabulados para obter os resultados. Assim, para tabulação dos dados e elaboração das tabelas, utilizouse a planilha eletrônica Microsoft Excel.

Para fins de análise, foram realizados cálculos da nota média, que é o "valor obtido somando todos os elementos de um conjunto e dividindo a soma pelo número de elementos" (MALHOTRA, 2012). Cabe salientar que este trabalho considerou satisfatória a nota média total igual ou superior a 4.00, demonstrando que os principais relatórios apresentam, frequentemente ou sempre, as características da informação valiosa.

\section{RESULTADOS E DISCUSSÃO}

Analisando os resultados obtidos com a aplicação do questionário de pesquisa, identificou-se, inicialmente, o perfil dos gerentes regionais de vendas, sendo que a maioria $(63,64 \%)$ possui de 36 a 40 anos, $27,27 \%$ de 41 a 50 anos e $9,09 \%$ de 31 a 35 anos. A busca pelo aprimoramento/conhecimento é ressaltada, pois $63,64 \%$ têm pós-graduação completa e $36,36 \%$ incompleta. Audy et al. (2007), salienta que o conhecimento envolve "[...] uma combinação de instintos, ideias, informações, regras e procedimentos que guiam ações e decisões".

Diante do percentual apresentado, pode-se destacar que os gerentes regionais de vendas possuem conhecimento para entender as informações e identificar o quanto são eficientes e eficazes para o processo. Eficiência, para Beal (2012), é a "relação entre os produtos gerados por um processo e os custos dos insumos empregados", e eficácia, segundo Bio (2008), é a "relação entre resultados pretendidos/resultados obtidos".

A pesquisa demonstrou que a indústria de bebidas possui gerentes regionais de vendas representando todas as regiões de atuação: $45,45 \%$ estão situados na região Metropolitana, $27,27 \%$ no Vale do Taquari, e 9,09\% no Sul, nas Missões e na Serra. Quanto ao tempo de atuação na empresa, 27,27\% trabalham de 1 a 3 anos, o mesmo percentual trabalha de 3 a 6 anos e mais de 9 anos, e 18,18\% de 6 a 9 
anos. Referente à utilização de recursos tecnológicos, $81,82 \%$ possuem um grande conhecimento e 9,09\% consideram muito grande e moderado. Com este resultado, comprovou-se que os gerentes possuem habilidade para trabalhar com os sistemas utilizados no nível tático, ou seja, o Sistema de Informação Gerencial (SIG) e o Sistema de Apoio à Decisão (SAD), abordados na fundamentação teórica.

Para Audy et al. (2007), o Sistema de Informação Gerencial (SIG) atende "em grande parte os gerentes de nível tático da organização na forma de relatórios que apresentam indicadores sobre o desempenho de uma determinada área", assim como o Sistema de Apoio à Decisão (SAD), que além da integração com outros sistemas, permite "realizar análises e simulações como forma de comparar o impacto de diferentes decisões" (AUDY et al., 2007).

Atendendo ao objetivo específico, em reunião realizada envolvendo a autora e o gestor da Área de Informações, identificou-se que trinta e dois relatórios são disponibilizados aos gerentes regionais de vendas. Destes, dois foram classificados como principais relatórios, por abrangerem informações gerenciais, e os demais complementarem essas informações.

As definições acima mencionadas foram apresentadas ao Gerente Estratégico de Mercado, que concordou com a escolha. Uma vez demonstrado que os gerentes regionais de vendas possuem grande conhecimento, tanto intelectual quanto tecnológico, acredita-se que os mesmos tenham competência para avaliar os dois principais relatórios quanto às características da informação valiosa. Para Helal et al. (2009), alguém é competente quando constitui, articula e mobiliza valores, conhecimentos e habilidades para uma atuação transformadora. A seguir, são demonstrados os resultados da referida avaliação.

Referente ao "Relatório A", é possível observar nas Tabelas 1 e 2, abaixo, que a característica da informação 6.9, denominada "Acessível (a informação deve ser facilmente acessível aos funcionários autorizados)", obteve a melhor nota média total $(4,64)$. Há as características da informação valiosa de no 6.2 , $6.3,6.5,6.6,6.7,6.8$ e 6.10 , que receberam nota média total igual ou superior a 4.00 , evidenciando que estas características são identificadas pelos gerentes regionais de vendas.

Tabela 1: Número de repostas e nota média total atribuída a cada característica da informação valiosa - 'Relatório A'.

\begin{tabular}{|c|c|c|c|c|c|c|}
\hline \multirow{2}{*}{ № da assertiva } & \multicolumn{5}{|c|}{ Nível com que apresenta cada característica da informação valiosa (Escala Likert) } & \multirow{2}{*}{$\begin{array}{c}\text { Nota média } \\
\text { total }\end{array}$} \\
\hline & Nunca (1) & Raramente (2) & Às vezes (3) & Frequentemente (4) & Sempre (5) & \\
\hline 6.1 & 0 & 1 & 3 & 6 & 1 & 3,64 \\
\hline 6.2 & 0 & 0 & 3 & 4 & 4 & 4,09 \\
\hline 6.3 & 0 & 1 & 2 & 2 & 6 & 4,18 \\
\hline 6.4 & 0 & 0 & 4 & 4 & 3 & 3,91 \\
\hline 6.5 & 0 & 0 & 1 & 4 & 6 & 4,45 \\
\hline 6.6 & 0 & 0 & 2 & 4 & 5 & 4,27 \\
\hline 6.7 & 0 & 0 & 2 & 2 & 7 & 4,45 \\
\hline 6.8 & 0 & 0 & 4 & 2 & 5 & 4,09 \\
\hline 6.9 & 0 & 0 & 1 & 2 & 8 & 4,64 \\
\hline 6.10 & 0 & 0 & 3 & 3 & 5 & 4,18 \\
\hline
\end{tabular}

A pesquisa revela que a característica da informação valiosa 6.1, denominada 'Preciso/Exato (a informação precisa/exata não contém erros)', teve a menor nota média total $(3,64)$, seguida da 6.4 , denominada 'Confiável (é a informação em que se pode acreditar, os dados de origem e os métodos de coleta 
de dados são confiáveis)', demonstrando que ambas não são identificadas pelos gerentes regionais de vendas.

Tabela 2: Nota média total de cada característica da informação valiosa - 'Relatório A'.

\begin{tabular}{|c|l|r|}
\hline $\begin{array}{c}\text { No da } \\
\text { assertiva }\end{array}$ & \multicolumn{1}{|c|}{ Assertiva } & $\begin{array}{c}\text { Nota média } \\
\text { total }\end{array}$ \\
\hline 6.9 & Acessível (a informação deve ser facilmente acessível aos funcionários autorizados). & 4,64 \\
\hline 6.5 & Relevante (a informação deve ser relevante para o tomador de decisão). & 4,45 \\
\hline 6.7 & Pontual (a informação pontual é aquela obtida e disponível quando necessária). & 4,45 \\
\hline 6.6 & Simples (a informação deve ser simples para facilitar a tomada de decisão). & 4,27 \\
\hline 6.3 & Flexível (a informação deve ser usada para diversos propósitos). & 4,18 \\
\hline 6.10 & Seguro (o acesso à informação deve ser permitido apenas aos funcionários autorizados). & 4,18 \\
\hline 6.2 & Completo (a informação completa contém todos os fatos importantes). & 4,09 \\
\hline 6.8 & $\begin{array}{l}\text { Verificável (significa que a informação pode ser conferida quando necessário, para se assegurar } \\
\text { que ela está correta). }\end{array}$ & 4,09 \\
\hline 6.4 & $\begin{array}{l}\text { Confiável (é a informação na qual se pode acreditar, os dados de origem e os métodos de coleta } \\
\text { de dados são confiáveis). }\end{array}$ & 3,91 \\
\hline 6.1 & Preciso/Exato (a informação precisa/exata não contém erros). & 3,64 \\
\hline
\end{tabular}

Tanto o 'Relatório A' como o 'Relatório B', analisado na sequência, foram avaliados quanto à frequência de recebimento, e 100,00\% dos respondentes consideraram que a frequência está de acordo com a necessidade de utilização. Quanto ao 'Relatório B', é possível identificar por meio das tabelas 3 e 4 , demonstradas abaixo, que as características da informação valiosa 8.5 e 8.7, denominadas respectivamente, 'Relevante (a informação deve ser relevante para o tomador de decisão)' e 'Pontual (a informação pontual é aquela obtida e disponível quando necessária)', apresentaram as melhores notas médias totais, ambas com 4,27 .

Tabela 3: Número de repostas e nota média total atribuída a cada característica da informação valiosa - 'Relatório B'.

\begin{tabular}{|c|c|c|c|c|c|c|}
\hline \multirow{2}{*}{ № da assertiva } & \multicolumn{5}{|c|}{ Nível com que apresenta cada característica da informação valiosa (Escala Likert) } & \multirow{2}{*}{ Nota média total } \\
\hline & Nunca (1) & Raramente (2) & Às vezes (3) & Frequentemente (4) & Sempre (5) & \\
\hline 8.1 & 0 & 2 & 4 & 3 & 2 & 3,45 \\
\hline 8.2 & 0 & 2 & 3 & 2 & 4 & 3,73 \\
\hline 8.3 & 0 & 2 & 2 & 2 & 5 & 3,91 \\
\hline 8.4 & 0 & 2 & 1 & 5 & 3 & 3,82 \\
\hline 8.5 & 0 & 1 & 0 & 5 & 5 & 4,27 \\
\hline 8.6 & 0 & 1 & 3 & 1 & 6 & 4,09 \\
\hline 8.7 & 0 & 1 & 1 & 3 & 6 & 4,27 \\
\hline 8.8 & 1 & 1 & 3 & 3 & 3 & 3,55 \\
\hline 8.9 & 0 & 1 & 2 & 2 & 6 & 4,18 \\
\hline 8.10 & 0 & 1 & 1 & 4 & 5 & 4,18 \\
\hline
\end{tabular}

Tabela 4: Nota média total de cada característica da informação valiosa - 'Relatório B'.

\begin{tabular}{|c|l|r|}
\hline $\begin{array}{c}\text { No da } \\
\text { assertiva }\end{array}$ & \multicolumn{1}{|c|}{ Assertiva } & $\begin{array}{c}\text { Nota média } \\
\text { total }\end{array}$ \\
\hline 8.5 & Relevante (a informação deve ser relevante para o tomador de decisão). & 4,27 \\
\hline 8.7 & Pontual (a informação pontual é aquela obtida e disponível quando necessária). & 4,27 \\
\hline 8.9 & Acessível (a informação deve ser facilmente acessível aos funcionários autorizados). & 4,18 \\
\hline 8.10 & Seguro (o acesso à informação deve ser permitido apenas aos funcionários autorizados). & 4,18 \\
\hline 8.6 & Simples (a informação deve ser simples para facilitar a tomada de decisão). & 4,09 \\
\hline 8.3 & Flexível (a informação deve ser usada para diversos propósitos). & 3,91 \\
\hline 8.4 & $\begin{array}{l}\text { Confiável (é a informação na qual se pode acreditar, os dados de origem e os métodos de coleta } \\
\text { de dados são confiáveis). }\end{array}$ & 3,82 \\
\hline 8.2 & Completo (a informação completa contém todos os fatos importantes). & 3,73 \\
\hline 8.8 & $\begin{array}{l}\text { Verificável (significa que a informação pode ser conferida quando necessário, para se assegurar } \\
\text { que ela está correta). }\end{array}$ & 3,55 \\
\hline 8.1 & Preciso/Exato (a informação precisa/exata não contém erros). & 3,45 \\
\hline
\end{tabular}


As características da informação valiosa de no 8.6, 8.9 e 8.10 conseguiram nota média total igual ou superior a 4,00, evidenciando que estas características são identificadas no 'Relatório B', segundo a percepção dos gerentes regionais de vendas. Verificou-se, também, que a característica da informação valiosa 8.1 teve a menor nota média total $(3,45)$, denominada 'Preciso/Exato (a informação precisa/exata não contém erros)', seguida das características da informação valiosa 8.2, 8.3, 8.4 e 8.8, que da mesma forma que a 8.1, não atingiram nota média total igual ou superior a 4,00, comprovando, assim, que não são identificadas pelos gerentes regionais de vendas no 'Relatório B'.

\section{CONSIDERAÇÕES FINAIS}

Com base neste estudo, evidencia-se o valor da informação para as organizações, pois permite, conforme Beal (2012), que as escolhas sejam feitas com maior segurança e no momento certo, e conforme Audy et al. (2007), que se possa identificar ameaças e oportunidades, criando um cenário para uma resposta eficaz e competitiva. Observou-se que das dez assertivas (6.1 a 6.10) referentes ao 'Relatório A', oito adquiriram nota média total entre 4,64 e 4,09; e duas obtiveram nota média total 3,91 e 3,64. Diante das notas médias totais apresentadas, conclui-se que o 'Relatório A', na percepção dos gerentes regionais de vendas, é eficiente e eficaz.

Contudo, das dez assertivas (8.1 a 8.10) referentes ao 'Relatório B', cinco apresentaram nota média total entre 4,27 e 4,09; e cinco receberam nota média total entre 3,91 e 3,45, assim, conclui-se que o "Relatório B", na percepção dos gerentes regionais de vendas, não é eficiente e eficaz, sendo necessária avaliação e possível reestruturação. Esse resultado evidencia a falta de gestão da informação, principalmente no 'Relatório B'. Conforme Beal (2012), a gestão da informação assegura o valor estratégico da informação, pois é um processo que consiste nas atividades de identificação, busca, classificação, tratamento, armazenamento e disseminação de informações.

Propõe-se, diante do cenário apresentado, a implementação de formas de conferência antes da disponibilização das informações, para assegurar que as mesmas sejam precisas/exatas, ou seja, não contenham erros e sejam confiáveis. Também sugere-se a utilização de recursos tecnológicos, visando a flexibilidade e otimização do tempo, ou seja, a redução do trabalho manual. Segundo Audy et al. (2007), por meio da utilização de sistemas de informação, as organizações melhoram sua gestão, automatizam os processos, integram áreas, clientes e fornecedores, disponibilizam informações confiáveis para todos os níveis organizacionais, evitam perda de informações, eliminam retrabalhos e reduzem custos.

E, por fim, sugere-se realizar levantamento das necessidades dos usuários, para disponibilizar as informações que realmente sejam úteis e no layout que atenda as expectativas dos mesmos. Concluindo, acredita-se que todos os objetivos desta pesquisa foram atendidos e que as iniciativas sugeridas podem auxiliar na busca permanente de melhor performance.

\section{REFERÊNCIAS}


BEAL, A.. Gestão estratégica da informação: como transformar a informação e a

BIO, S. R.. Sistemas de informação: um enfoque gerencial. 2 ed. São Paulo: Atlas, 2008.

BOTELHO, D.; ZOUAIN, D. M.. Pesquisa quantitativa em administração. São Paulo: Atlas, 2006.

GIL, A. C.. Como elaborar projetos de pesquisa. 5 ed. São Paulo: Atlas, 2010.

GIL, A. C.. Métodos e técnicas de pesquisa social. 6 ed. São Paulo: Atlas, 2011.

HELAL, D. H.; GARCIA, F. C.; HONÓRIO, L. C.. Gestão de pessoas e competência: teoria e pesquisa. Curitiba: Juruá, 2009.

MALHOTRA, N. K.. Pesquisa de marketing: uma orientação aplicada. 4 ed. Porto Alegre: Bookman, 2006.
MALHOTRA, N. K.. Pesquisa de marketing: uma orientação aplicada. 6 ed. Porto Alegre: Bookman, 2012.

MARCONI, M. A.; LAKATOS, E. M.. Fundamentos de metodologia científica. 7 ed. São Paulo: Atlas, 2010.

REYNOLDS, G. W.; STAIR, R. M.. Princípios de sistemas de informação. 9 ed. São Paulo: Cengage Learning, 2011.

ROESCH, S. M. A.. Projetos de estágios e de pesquisa em administração: guia para estágios, trabalhos de conclusão, dissertações e estudos de caso. 3 ed. São Paulo: Atlas S.A., 2013.

VERGARA, S. C.. Métodos de pesquisa em administração. 4 ed. São Paulo: Atlas S.A., 2010.

VERGARA, S. C.. Projetos e relatórios de pesquisa em Administração. 7 ed. São Paulo: Atlas, 2006.

VERGARA, S. C.. Projetos e relatórios de pesquisa em administração. 12 ed. São Paulo: Atlas, 2010. 